\title{
Labyrinthe
}

15 | 2003

Territoires : questions d'images

\section{De la recherche à l'action: les passions urbaines d'un libraire}

\section{Xavier Capodano}

\section{(2) OpenEdition}

12 Journals

Édition électronique

URL : http://journals.openedition.org/labyrinthe/476

DOI : $10.4000 /$ labyrinthe.476

ISSN : 1950-6031

Éditeur

Hermann

Édition imprimée

Date de publication : 1 juin 2003

\section{Référence électronique}

Xavier Capodano, « De la recherche à l'action: les passions urbaines d'un libraire », Labyrinthe [En ligne], 15 | 2003, mis en ligne le 25 juillet 2008, consulté le 22 avril 2019. URL : http:// journals.openedition.org/labyrinthe/476 ; DOI : 10.4000/labyrinthe.476

Ce document a été généré automatiquement le 22 avril 2019.

Propriété intellectuelle 


\title{
De la recherche à l'action: les passions urbaines d'un libraire
}

\author{
Xavier Capodano
}

Entretien réalisé en mars 2003 par Laurence Marie et Clément Orillard du comité de rédaction de la revue Labyrinthe ${ }^{1}$.

2 Qu'aimons-nous dans les villes, si ce n'est l'étonnement, la surprise et les fructueuses rencontres qu'elles nous procurent?

3 En ce début de printemps, le soleil réchauffant l'air chargé de cris et d'effluves qui évoquent l'Extrême-Orient, les débuts de la rue de Belleville, laborieuse comme toujours, offrent l'image chatoyante et pittoresque d'un classique chinatown en plein développement. La surprise est grande lorsque le flâneur, porté par ses pas, décide d'explorer l'envers du décor et découvre un univers résolument différent, conservatoire de la mémoire du quartier, cadavre exquis de l'urbanisme où perdurent les marques d'une identité juive et arabe.

4 Entre ces deux mondes, encadrée par un squat d'artistes et une soupe populaire, une devanture blanche, fraîchement peinte, surprend. L'enseigne, Le Genre urbain, s'offre telle une méditation sur son environnement. De larges vitrines confirment ce qui n'était encore qu'un soupçon : c'est une librairie, commerce inhabituel dans ce quartier.

5 À l'intérieur, porté par l'ambiance chaleureuse, le visiteur se retrouve immergé au sein d'une littérature variée, des ouvrages scientifiques aux romans en passant par des recueils de photographies ou des albums d'illustration, tous consacrés au même thème : la ville, bien sûr. Devant l'abondance, le regard hésite, le doigt court sur les couvertures. Intervient opportunément le maître des lieux: «Puis-je vous aider?» Passionné, intarissable, il commence par la présentation de divers ouvrages pour glisser peu à peu vers une discussion plus générale et plus animée autour des questions urbaines. Finalement, l'homme se présente. «Je m'appelle Xavier Capodano, j'ai ouvert récemment cette librairie avec ma femme. Avant j'étais sociologue.»Et la discussion de se poursuivre... 
6 Labyrinthe - Votre parcours personnel est plutôt atypique. Tout d'abord, pour quelle raison, après un BTS action commerciale, vous êtes-vous lancé dans une thèse de sociologie?

7 Xavier Capodano - Après avoir passé mon BTS, je me suis vite rendu compte que je n'étais pas fait pour le commerce et j'ai eu envie de commencer des études universitaires. J'ai alors choisi la sociologie un peu par hasard, pour rassurer mes parents et aussi parce que j'espérais devenir un meilleur commercial en m'appuyant sur les méthodes bourdieusiennes. Mais je me suis pris au jeu et je suis allé jusqu'au DEA. Puis, pour tromper mon ennui pendant le service militaire, j'ai rempli un énorme dossier de candidature à un appel d'offre de l'Agence de l'environnement et de la maîtrise de l'énergie. Et j'ai décroché une bourse de thèse.

\section{Pourquoi avoir choisi de vous spécialiser en sociologie urbaine ?}

9 Parce que je suis un citadin pur et dur. J'adore Paris, que je connais d'ailleurs encore mieux depuis que je fais mes tournées de libraire en scooter. Après une enfance passée dans une cité HLM de l'Essonne, j'ai déménagé dans le XIII ${ }^{\mathrm{e}}$ arrondissement avec mes parents, pieds-noirs d'origine. En 1976, le quartier était un vaste chantier. J'ai vu de près ses mutations, puisque je passais mon temps dans la rue, au point que j'ai mal tourné et que j'ai été renvoyé de l'école. Vers l'âge de douze ou treize ans, j'ai appris à aimer cette ville en l'observant par " capillarité », c'est-à-dire en étendant peu à peu mon périmètre d'exploration.

Vos activités de recherche ont-elles satisfait votre curiosité initiale à l'égard de l'espace urbain?

11 C'est ce que j'espérais au départ, mais j'ai assez vite compris que l'univers de la recherche ne me convenait pas. J'ai commencé par travailler au laboratoire Lires à l'École normale supérieure de Cachan, avant de suivre ma directrice de thèse au Centre de recherche sur l'habitat. Parallèlement à mes recherches, j'enseignais la sociologie générale à la fac et dans un institut pour travailleurs sociaux. Ce travail passionnant m'a permis de me spécialiser dans les questions urbaines et sociales. Mais ma thèse s'est quant à elle beaucoup moins bien passée. J'ai d'abord eu des difficultés à trouver ma place au sein du laboratoire de recherche, notamment en raison de problèmes relationnels avec ma directrice. J'ai abandonné mes recherches une première fois. Puis, après un retour enthousiaste, je me suis rendu compte que, même si je terminais ma thèse, j'aurais le plus grand mal à décrocher un poste en région parisienne. J’ai alors décidé de tout arrêter.

Votre expérience de la recherche institutionnelle semble vous avoir laissé un goût amer. Quel regard portez-vous aujourd'hui sur elle?

13 Ma position est un peu contradictoire. D'un côté, je pense que la question urbaine doit être éclairée par la recherche fondamentale et qu'il est donc nécessaire de maintenir une sorte de bulle coupée du monde, hors de toute logique utilitariste suscitée par une demande sociale à court terme. Il faut laisser aux chercheurs le temps d'approfondir leurs analyses, même s'ils ont aussi besoin d'être secoués de temps en temps : les paresseux sont nombreux dans ce milieu. D'un autre côté, je regrette que la recherche soit dans une logique d'hyperspécialisation, qui s'appuie sur le constat erroné que tout a déjà été étudié. La plupart du temps, les chercheurs ne puisent leurs sources que dans les travaux de leurs homologues et délaissent l'éclairage apporté par le terrain et les autres disciplines, notamment la sociologie. C'est pour cette raison que, paradoxalement, les travaux de recherche ne nous apprennent souvent pas grand-chose. 

Le fait d'avoir passé plus de temps à étudier la sociologie qu'à faire de la recherche a changé mon regard sur la société. D'une part, la sociologie est un outil de déconstruction du réel qui permet de décoder les discours, les pratiques et les institutions. Ce décryptage est essentiel pour le sociologue de l'urbain, puisqu'il s'occupe avant tout des acteurs du territoire. J'ai d'ailleurs été étonné que mes anciens collègues du laboratoire n'aient qu'une connaissance diffuse des pères de la sociologie. D'autre part, étudier cette discipline m'a donné envie de me dégager de la mécanique sociale décrite par Bourdieu. Venant moi-même d'un milieu populaire, je me suis senti escroqué par les études universitaires, car le système est verrouillé par les principes de cooptation et la priorité donnée aux élèves des grandes écoles. En d'autres termes, les diplômants dans mon cas aspirent à un milieu qui les méprise et veulent se dégager d'un milieu qu'ils ne comprennent plus. C'est à ce moment-là que l'on reproduit de façon aigrie le modèle parental à un niveau supérieur. J'ai donc choisi de prendre une autre voie, en espérant que tous ces gens qui me méprisent viendront à moi et que les autres comprendront mon projet.

16 Comment avez-vous décidé de changer de vie et de créer une librairie spécialisée dans les questions urbaines?

17 L'idée a fait son chemin en plusieurs étapes. Après avoir abandonné mes recherches, j'ai travaillé comme conseiller scientifique à l'Institut d'économie urbaine. Mais je trouvais frustrant de ne pas passer moi-même à l'action. Je voulais m'investir dans un territoire, et pas seulement en collaborant à des études ou à des causeries. Au cours de mes recherches, j'ai passé un an à observer les quartiers en difficulté d'Aubervilliers, ce qui m'a donné des idées sur les actions concrètes à mener. J'ai d'abord voulu créer une revue sur la ville, mais le copain éditeur avec lequel je voulais la monter a claqué la porte de sa maison d'édition et m'a laissé avec ma revue sur les bras. J'ai alors réfléchi à un projet pendant près d'un an. Au départ, je voulais créer un centre de ressources réunissant des chercheurs et des habitants, une sorte de plate-forme ouverte sur la ville qui aurait permis de mieux comprendre l'environnement urbain. Mais le nombre d'associations était déjà très élevé. Un projet économique me paraissait plus viable. Le copain avec qui je voulais m'associer au départ m'a conseillé d'ouvrir une librairie d'urbanisme, ce que j'ai finalement fait, avec ma femme, et sans lui, car il a préféré se consacrer à l'écriture.

Comment définiriez-vous votre démarche par rapport à celle du Moniteur, la grande librairie parisienne d'architecture et d'urbanisme?

19 Jusqu'à présent, Le Moniteur était effectivement la seule librairie parisienne spécialisée dans l'urbain. Mais notre démarche n'est pas la même. Le Moniteur est un groupe d'éditeurs financé par Vivendi, alors que moi, je suis tout seul. De plus, il est avant tout destiné aux spécialistes et aux gens aisés, tandis que ma librairie privilégie l'approche de la ville par le citadin : elle s'adresse à tous ceux qui s'intéressent à ce qui se passe autour d'eux, et donc pas seulement à la clientèle du Moniteur. Durkheim, Elias et Bourdieu l'ont d'ailleurs bien montré. Un lieu commun tenace fait de la ville un espace répulsif parce que anonyme. Pourtant, c'est précisément cet anonymat que bien des gens recherchent en venant y habiter. Entre la vision de la ville véhiculée 
par les journaux - qui ne parlent que des quartiers réputés dangereux ou de la flambée des prix de l'immobilier - et les Annales, il existe des milliers d'aspects que j'aimerais mettre en valeur. Je cherche à opérer une connexion entre toutes les littératures sur la ville, en essayant de remplir chaque interstice potentiel : la littérature, la photographie, les livres pour la jeunesse, l'illustration, la bande dessinée...

Pourquoi avoir choisi d'accorder aux œuvres de fiction une place équivalente à celle de la littérature de recherche au sein de votre rayon consacré à la ville ?

J'ai délibérément voulu faire la part belle à l'imaginaire dans les ouvrages que je propose. Ce choix est lié à ma prise de distance avec la recherche, laquelle est mal en point à mon avis, justement parce qu'elle a laissé l'imaginaire de côté. Les œuvres de fiction comme $L a$ Forme d'une ville de Julien Gracq, ou Espèces d'espaces de Georges Perec retraduisent le territoire urbain dans une langue qui nous ouvre les yeux et nous montre les territoires comme on a oublié de les voir. Il est plus facile de comprendre la déshérence de certains quartiers en lisant Zones de Jean Rolin ou les polars de Donald Westlake qu'en épluchant un manuel de procédures. Et l'on perçoit mieux les logiques territoriales et l'idée de « non-ville », de zone intermédiaire entre le centre ville et la banlieue, après s'être plongé dans La Clôture de Jean Rolin. L'important, c'est la façon dont ce qu'on lit nous permet de nous projeter dans la réalité.

Un des principaux points faibles de la recherche urbanistique serait donc son manque d'ouverture à l'imaginaire?

C'est ce que je crois en effet. Prenons un exemple. Dans Extension du domaine de la lutte, Houellebecq montre la misère humaine des personnes qui partent suivre des stages de formation en province, leur solitude dans leur chambre d'hôtel, les relations éphémères qu'ils nouent. Jamais un chercheur n'oserait faire cela : il lui faudrait d'abord verrouiller ses sources, effectuer une étude de terrain, puis confronter les points de vue. En cinq pages, Houellebecq nous en apprend davantage sur les relations sociales qui se développent au sein de l'espace urbain qu'un chercheur en un énorme pavé indigeste. Mais soyons juste: il arrive aussi que la recherche ouvre sur l'imaginaire. Dans Paris mosaïque : promenades urbaines, les sociologues Michel Pinçon et Monique Pinçon-Charlot nous emmènent à la découverte concrète de la ville, tout en définissant clairement les outils statistiques et scientifiques qu'ils ont utilisé pour leur méthode d'observation. Dans Voyage en grande bourgeoisie, ils montrent comment la position sociale a des conséquences sur l'espace, et, réciproquement, comment les espaces très marqués socialement conditionnent les pratiques des habitants. Eux aussi, à l'instar des romanciers, nous fournissent d'excellentes lunettes sur le monde qui nous entoure.

Pourquoi vous être vous-même implanté au sein d'un espace socialement marqué : dans le $\mathrm{xx}^{\mathrm{e}}$ arrondissement, à deux pas de la rue de Belleville où se côtoient populations chinoise et arabe ?

27 J'habite à Belleville depuis longtemps, et je m'y plais. C'est ici que j'avais envie de m'installer, même si c'est un quartier difficile - d'ailleurs, nous avons été récemment victimes d'un cambriolage. Et puis Belleville est une zone subventionnée par un Fond de revitalisation économique, destiné à redensifier le tissu économique par des activités commerciales.

\section{Avez-vous été soutenus par les milieux institutionnels ?}

29 Pas vraiment. Nous avons eu des difficultés à obtenir des financements, notamment à cause de la standardisation du regard des institutions. Par exemple, nous n'avons pas 
décroché de subvention en première commission du Comité national des lettres, sous le motif que les membres du comité ne nous connaissaient pas. Et lorsque la responsable est finalement venue nous voir suite à notre invitation, elle nous a conseillé de décorer la librairie en nous inspirant des différentes nationalités de Belleville. Encore une façon d'essayer de nous resituer dans un cadre bien précis. Nous avons bien entendu refusé.

\section{Vous considérez-vous comme un libraire « activiste » ?}

Pas dans le sens où on l'entend généralement. Je refuse le militantisme béat. Mon projet n'est pas militant, mais cohérent : il est le fruit de mon parcours et de mon expérience. J'ai une fibre populaire et je suis aussi un peu un intello; c'est pourquoi je pense que les quartiers populaires ont droit à un projet intellectuel et que tout le monde a sa place dans Le Genre urbain. Après réflexion, nous avons donc choisi de ne pas seulement être une librairie spécialisée. Nous sommes donc aussi une librairie de quartier qui propose des ouvrages généraux et passe commande des livres dont nos clients ont besoin. J'essaie de me mettre au diapason de Belleville et de recréer un lieu de vie qui profite à la fois aux habitants et à nous-mêmes. Après tout, c'est la fonction que remplit le boulanger : on a besoin de lui et lui, de son côté, il fédère les habitants du quartier lorsqu'ils papotent en faisant la queue pour acheter du pain. Personne n'ira lui reprocher d'être un sale capitaliste. Petit à petit, les habitants commencent à nous identifier, et les chercheurs à nous rendre visite, comme Jean-Paul Flamand, historien du logement social et Edmond Préteceille, sociologue spécialiste des questions de ségrégation urbaine. Je préfère discuter avec eux dans ma librairie plutôt que dans un laboratoire. Pour ma femme et moi, Le Genre urbain est bien plus qu'une librairie : c'est un lieu de vie.

\section{Quel rapport entretenez-vous avec les associations de Belleville?}

Notre projet a d'abord été un peu méprisé par les associations du quartier, puis la glace a fondu assez rapidement. De toute façon, je refuse de travailler avec les associations sociales car je suis en désaccord avec leur vision de la pauvreté et des acteurs. Elles se veulent représentatives du quartier par les actions qu'elles y mènent mais elles l'ont figé en en faisant une stratification d'associations et en refusant des projets porteurs qui ne s'alignaient pas sur leur politique. De plus, favoriser à tout prix comme elles le font la mixité des cultures au sein du même immeuble conduit à la catastrophe. Alors que, au contraire, il est possible à des cultures différentes de cohabiter dans la même rue et pas forcément dans le même bâtiment. On ne peut malheureusement pas empêcher le quartier de Belleville de s'embourgeoiser peu à peu. Et justement, je pense qu'on a été cambriolés parce qu'on renvoie cette image d'envahisseurs.

\section{Avez-vous prévu de développer des activités sur le thème de la ville ?}

Nous préparons de petites animations, notamment avec Luc Baboulet, qui est architecte et enseignant. Beaucoup de choses ne peuvent se dire dans les colloques. J'aimerais justement que ma librairie soit un des lieux de vie des questions urbanistiques, que s'y exprime une parole plus vivante et plus engagée, hors institution, et que les spécialistes puissent échanger leurs idées directement avec la population. Et pour cela il faut des gens qui nous ressemblent, et pas exclusivement des chercheurs. À plus long terme, j'aimerais éditer des textes portant sur la ville. Globalement, j'ai vocation à ce que Le Genre urbain soit pour les auteurs, sinon un tremplin, du moins une plate-forme. 


\section{ÉLÉMENTS BIBLIOGRAPHIQUES}

Houellebecq Michel, Extension du domaine de la lutte, Paris, Maurice Nadeau, 1994.

Gracq Julien, La Forme d'une ville, Paris, José Corti, 1985.

Perec Georges, Espèces d'espaces, Paris, éditions Galilée, 1974.

Pinçon Michel \& Pinçon-Charlot Monique, Voyage en grande bourgeoisie, Paris, Puf, 1998.

-, Paris mosaïque : promenades urbaines, Paris, Calmann-Lévy, 2001. Rolin Jean, Zones, Paris, Gallimard, 1995.

-, La Clôture, Paris, P.O.L, 2001. Westlake, Donald E., Château en esbroufe, Paris, Gallimard, 1981.

$42-$, Le Couperet, Paris, Payot \& Rivages, 1998.

\section{NOTES}

1. Sociologue de la ville de formation et créateur de la librairie Le Genre urbain, Xavier Capodano est né en 1967. Après un baccalauréat professionnel, il obtient en 1988 un BTS action commerciale et s'oriente ensuite vers la sociologie à l'université Paris X-Nanterre. En 1992, il soutient une maîtrise de sociologie, option développement économique et social, puis un DEA de sciences sociales co-habilité par l'ENS Cachan. Son expérience de la sociologie est très variée : il a notamment été expert pour des organismes publics et enseignant en premier cycle universitaire. Spécialisé en sociologie urbaine, il a publié notamment "Dire les nouveaux territoires: du stigmate de la banlieue à l'ubiquité du paysage » avec Germain Adell, dans Nommer les nouveaux territoires urbains, Paris, éditions de l'Unesco-Fondation Maison des sciences de l'homme, 2001. Il a quitté le laboratoire CHR-Louest pour fonder la librairie Le Genre urbain avec sa femme, Sophie Manceau de Lafitte, tout en poursuivant son activité d'enseignement 